\title{
Modélisation élastoplastique endommageable des composites
}

\author{
Frédéric Lachaud ${ }^{1, a}$, Eric Haramburd ${ }^{1}$ et Philippe Jetteur ${ }^{2}$ \\ 1 École Nationale Supérieure d’Ingénieurs de Constructions Aéronautiques, Département de Génie Mécanique, \\ Membre de l'Institut de Génie Mécanique de Toulouse, 1 place Émile Blouin, 31056 Toulouse, France \\ 2 SAMTECH Liège, Parc Scientifique du Sart-Tilman, Rue des chasseurs-ardennais, 4031 Liège (Angleur), Belgique
}

Reçu le 27 juillet 2004 ; accepté le 31 mai 2005

\begin{abstract}
Résumé - L'objectif de cette étude est de comprendre et de modéliser le comportement mécanique de divers types de composites (fibres courtes, fibres longues...) par le couplage d'un modèle micro-mécanique et d'un modèle d'endommagement. Le modèle micromécanique permet de déterminer les caractéristiques élastiques du matériau à partir des caractéristiques thermo-mécaniques de la fibre et de la matrice par une technique d'homogénéisation micromécanique. Le modèle d'endommagement, par l'utilisation du couplage plasticité à écrouissage isotrope et/ou cinématique non-linéaire avec l'endommagement, permet la prise en compte de l'évolution des déformations résiduelles et de l'augmentation de la pseudo-limite élastique au cours du chargement
\end{abstract}

Mots clés : Composites / endommagement / plasticité / éléments finis / SMC / homogénéisation

\begin{abstract}
Elastoplastic damage modelling of composite materials. The objective of this work is to understand and model the mechanical behavior of various types of composites materials (short fibres, laminates...) by the coupling of a micromechanical model and a damage model. The micromechanical model makes it possible to determine the elastic characteristics of material starting from the data on the fibers and the matrix by a homogenization technique. The damage model by the use of the coupling between isotropic and/or non-linear kinematic hardening with the damage, allows taken into account of the development of the residual strains and the increase of the pseudo-yield stress during the loading.
\end{abstract}

Key words: Composites / damage / plasticity / finite element method / SMC / homogeneization

\section{Introduction}

La fiabilité des structures composites doit passer par une meilleure fabrication couplée à l'utilisation de matériaux nouveaux de plus en plus performants, mais aussi par une meilleure compréhension de leur comportement. La prévision de l'endommagement avant rupture, son influence sur la rupture, l'amélioration des critères de rupture sont autant de thèmes à développer pour une prédiction plus fiable du comportement des structures, autant sous sollicitations statiques que dynamiques.

Dans cette étude, il est proposé un modèle d'endommagement permettant une meilleure prédiction du comportement des structures composites. Deux matériaux sont utilisés. Le premier est un composite à fibres de verre courtes plutôt utilisé dans l'industrie automobile, le second est un composite stratifié carbone/époxy très utilisé dans l'industrie aéronautique. Les lois présentées

\footnotetext{
a Auteur correspondant : Frederic.Lachaud@ensica.fr
}

sont toutes implantées dans un code industriel de calculs par éléments finis.

\section{Expérimentation}

\subsection{Les matériaux étudiés}

Les composites étudiés sont de deux types :

1) Un mat de verre préimprégné SMC (Sheet Molding Compound) composé d'un mélange de fibres de verre courtes, de résine polyester insaturée et de charges minérales. Il est désigné par SMC-R 40 indiquant que les fibres courtes $(30 \mathrm{~mm})$ sont disposées aléatoirement (dans le plan de fabrication des plaques) et que le taux massique de fibres est de $40 \%$. Ainsi, la disposition aléatoire des fibres de ce composite lui confère un comportement isotrope transverse dans le plan $(X Y)$. Sa composite massique est résumée ci-dessous (MENZOLIT) :

- $40 \%$ de fibres de verre E, 


\section{Nomenclature}

\begin{tabular}{|lll|}
\hline$A_{\mathrm{F}}$ & Tenseur de localisation de Ferrari & $\mathrm{MPa}$ \\
$A_{\mathrm{MT}}$ & Tenseur de localisation de Mori et Tanaka & $\mathrm{MPa}$ \\
$C_{\mathrm{f}}$ & Tenseur de rigidité de la fibre & $\mathrm{MPa}$ \\
$C_{\mathrm{m}}$ & Tenseur de rigidité de la matrice & $\mathrm{MPa}$ \\
$d_{i}$ & Variable scalaire d'endommagement & \\
$\varepsilon^{\mathrm{e}}$ & Tenseur de déformation élastique & \\
$\varepsilon^{\mathrm{p}}$ & Tenseur de déformation plastique & \\
$E_{\mathrm{Esh}}$ & Tenseur d'Eshelby & $\mathrm{MPa}$ \\
$f$ & Critère de plasticité & $\mathrm{MPa}$ \\
$\lambda$ & Multiplicateur plastique & \\
$p$ & Déformation plastique cumulée \\
$R$ & Variable d'écrouissage isotrope & $\mathrm{MPa}$ \\
$R_{0}$ & Limite élastique & $\mathrm{MPa}$ \\
$\sigma$ & Tenseur de contrainte & $\mathrm{MPa}$ \\
$\tilde{\sigma}$ & Tenseur de contrainte effective & $\mathrm{MPa}$ \\
$V_{\mathrm{f}}$ & Taux volumique de fibres & \\
$X$ & Variable tensorielle d'écrouissage cinématique & $\mathrm{MPa}$ \\
$Y_{\mathrm{di}}$ & Taux de restitution d'énergie élastique & $\mathrm{MPa}$ \\
\hline
\end{tabular}

- $34 \%$ de charges minérales de trihydrate d'aluminium $\left(\mathrm{Al}\{\mathrm{OH}\}_{3}\right)$,

- 3,5\% de thermoplastique pour le retrait,

- 27,5\% de résine polyester insaturée.

La présence, dans nos échantillons, de trihydrate d'aluminium au lieu de carbonate de calcium généralement utilisé, confère au matériau des propriétés en température très importantes (augmentation de la température de transition vitreuse).

2) Un composite carbone-époxy constitué d'une nappe unidirectionnelle préimprégnée. Sa composition massique est résumée ci-dessous :

- $63 \%$ de fibres de carbone T2H,

- $34 \%$ de résine époxyde EH25 chargée de nodules de thermoplastique,

$-2 \%$ de porosité,

- la masse surfacique du pré-imprégné est de 260 g.m².

La nappe unidirectionnelle possède un comportement isotrope transverse dans le plan $(X Z)$.

Une dissolution à l'acide sulfurique réalisée selon la norme IGC 04-26-230 a été réalisée afin de connaître les pourcentages volumiques des fibres, de la résine, des charges et des porosités pour chaque matériau.

\subsection{Essais expérimentaux}

Afin de connaître le comportement de ce type de composite, des essais de traction, traction cyclée, ont été réalisés à l'aide d'une machine de traction INSTRON à déplacement imposé $\left(2 \mathrm{~mm} \cdot \mathrm{min}^{-1}\right)$. Les figures 1 et 2 montrent un exemple du comportement $\sigma=f(\varepsilon)$ pour le composite SMC-R40 et le composite stratifié carbone/époxy.

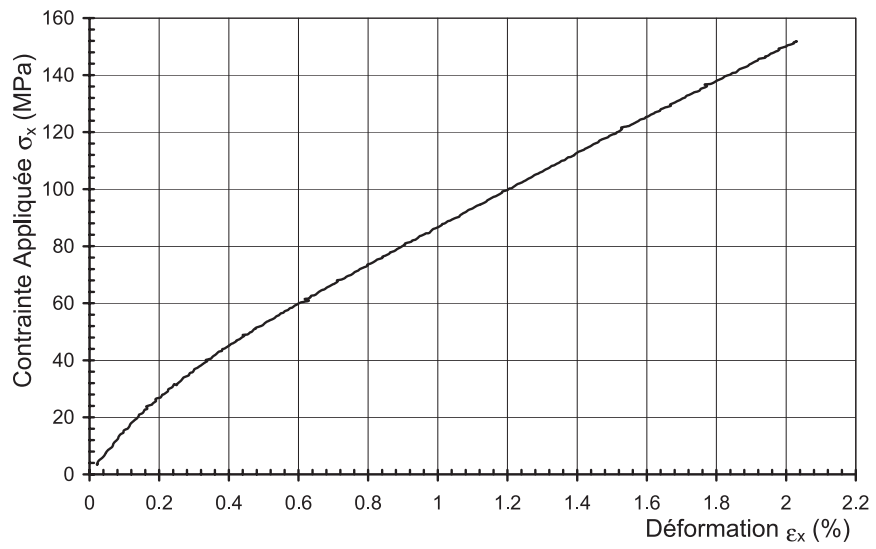

Fig. 1. Comportement contrainte-déformation longitudinale du SMC-R à $20^{\circ} \mathrm{C}$.

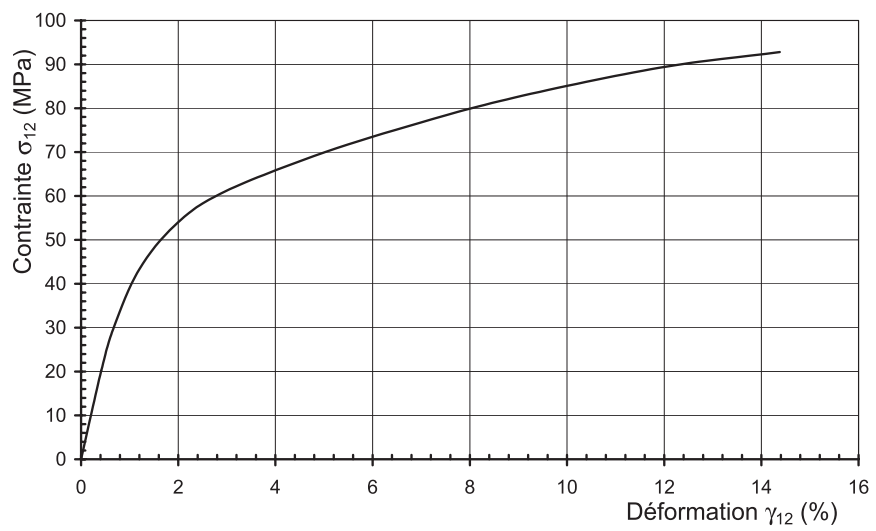

Fig. 2. Comportement d'un composite carbone-époxy $\left[ \pm 45^{\circ}\right]_{2 \mathrm{~S}}$.

\section{Modèle théorique de comportement}

Les techniques d'homogénéisation consistent à déterminer les propriétés élastiques d'un matériau à partir de ses constituants. Cette technique est réalisée 
sur un volume élémentaire représentatif $[1,2]$ permettant de prendre en compte l'ensemble des hétérogénéités. Par la suite, la reconstruction des grandeurs de l'échelle micro à l'échelle macro est réalisée par des modèles spécifiques. Dans le cadre de notre étude, la première étape consiste à déterminer les grandeurs homogénéisées de notre matériau à partir de ses constituants c'est-à-dire, de la fibre, de la résine polyester, des charges et des porosités.

Pour ce faire, nous avons abordé cette étape par une approche micromécanique réalisée en trois étapes : la représentation, la localisation, l'homogénéisation.

Cette approche a été réalisée par le modèle de Mori et Tanaka [3] couplé au modèle de l'inclusion équivalente d'Eshelby [4]. On rappelle ci-dessous le principe du modèle utilisé sachant que ce modèle a été utilisé sans modification. Bien que ce modèle soit généralement utilisé sur des composites à fibres courtes et pour des taux volumiques de fibres faibles (< à $30 \%$ ), nous l'avons tout de même utilisé pour des composites à fibres longues.

\subsection{Modèle micromécanique}

Les approches micromécaniques sont fondées sur le principe de l'homogénéisation et permettent de déterminer les propriétés macroscopiques d'un matériau hétérogène à partir des propriétés des différentes phases qui le constituent. Un volume élémentaire représentatif (VER) est défini. Les différentes caractéristiques y seront calculées.

\section{La représentation}

Dans notre étape de représentation du VER (Fig. 3), le composite SMC est constitué de nombreuses familles (une famille par orientation) de fibres de verre courtes (supposées de forme elliptique) noyées dans la matrice polyester. Les fibres sont réparties dans le plan de manière aléatoire. Pour représenter cette distribution, et compte tenu du comportement isotrope transverse dans le plan des plaques testées, la distribution aléatoire des fibres est représentée par vingt familles de fibres orientées de $0^{\circ}$ à $180^{\circ}[5]$.

Pour le composite unidirectionnel, les fibres de longueurs infinies, sont toutes orientées dans le même sens et constituent la couche. Toutes les grandeurs désignant les fibres sont notées avec l'indice i, celles correspondant à la matrice m. La phase notée i est l'ensemble des fibres de même orientation. Dans le cas du composite unidirectionnel, seule une famille de fibre est considérée. Le comportement global de l'empilement des couches est déterminé par la théorie classique des stratifiés [6].

\section{Les tenseurs de localisation/concentration}

La localisation (concentration) représente le lien entre les grandeurs macroscopiques et les grandeurs microscopiques (contrainte et déformation locales supposées

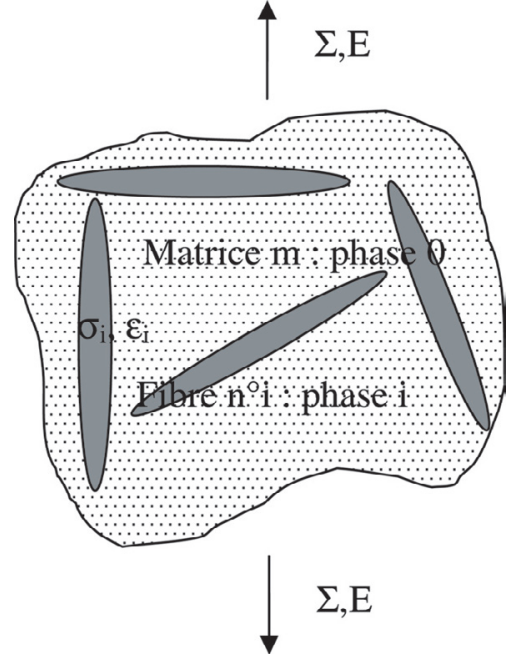

Fig. 3. Schéma du VER.

moyennées dans chaque phase). Elle fait intervenir le tenseur de localisation $A_{\mathrm{i}}$ pour un problème écrit en déformation et le tenseur $B_{\mathrm{i}}$ pour un problème écrit en contrainte :

$$
\left\{\begin{array}{l}
\sigma_{\mathrm{i}}=B_{\mathrm{i}} \cdot \Sigma \\
\varepsilon_{\mathrm{i}}=A_{\mathrm{i}} \cdot E
\end{array}\right.
$$

où $E$ et $\sum$ sont respectivement les déformations et contraintes macroscopiques et $\varepsilon_{\mathrm{i}}$ et $\sigma_{\mathrm{i}}$ sont respectivement les déformations et contraintes dans la phase $i$. La détermination de $A_{\mathrm{i}}$ et $B_{\mathrm{i}}$ dépend du modèle utilisé établi suivant la modélisation du comportement de chaque phase $[1,7]$.

\section{Modèle à base d'inclusions, problème d'Eshelby [4]}

Le problème de l'inclusion se base sur la situation élémentaire d'un milieu infini (matrice) perturbée localement par la présence d'une zone appelée inclusion $[2,7,8]$.

Le problème d'Eshelby (problème de l'inclusion homogène) fait référence à un ensemble homogène en terme de rigidité, entre la matrice et l'inclusion. Si l'inclusion subit une déformation «bloquée » ou « restreinte » par la matrice, il apparaît une contrainte non-nulle dans l'inclusion. La matrice réagissant à la contrainte dans l'inclusion en se déformant, il s'établit un état d'équilibre dans lequel l'inclusion et la matrice sont dans un état de contraintes (déformations homogènes non-nulles). Eshelby montre alors que la relation entre la déformation de l'inclusion et la déformation de perturbation liée à la forme de l'inclusion (ici elliptique), est fonction du tenseur d'ordre 4 noté $E_{\mathrm{Esh}}$ [4]. La contrainte dans l'inclusion est alors fonction de la rigidité de la matrice, du tenseur d'Eshelby et de la déformation dans l'inclusion. Si maintenant l'inclusion est inhomogène (rigidité différente de celle de la matrice), et plongée dans une matrice infinie, la solution du problème d'équilibre n'est autre que la superposition de la solution d'Eshelby et du chargement à l'infini (solution linéaire élastique). On parle alors de l'inclusion homogène équivalente. 
Eshelby donne alors, dans le cas où l'inclusion plongée dans la matrice joue le rôle d'une inclusion indépendamment des autres phases, la loi de localisation de la phase $i[2,4,5]$ donnée par l'équation (3).

\section{Modèle de Mori-Tanaka [2, 9-11]}

Cette théorie dite concentrée, permet de prendre en compte la présence d'un grand nombre d'hétérogénéités, constituant une amélioration du modèle d'Eshelby. Il existe des interactions locales entre chaque phase. La matrice dans laquelle est plongée l'hétérogénéité (l'inclusion) correspond à un milieu déjà perturbé par la présence des autres hétérogénéités.

Le tenseur de localisation de Mori et Tanaka (noté $A_{\mathrm{MT}}$ ) de la phase $i$ (fibre i) est donné par l'expression suivante :

$$
A_{\mathrm{MT}}^{i}=A_{\mathrm{Esh}}^{i}\left[\left(V_{\mathrm{f}} \cdot I+A_{\mathrm{Esh}}\right]^{-1}\right.
$$

Ce tenseur de localisation est fonction du tenseur de localisation d'Eshelby et noté $\left(A_{\text {Esh }}\right)$ :

$$
A_{\mathrm{Esh}}=\sum_{i=1}^{n} V_{\mathrm{f}}^{i} A_{\mathrm{Esh}}^{i}
$$

où

$$
A_{\mathrm{Esh}}^{i}=\left[I+E_{\mathrm{Esh}} S_{\mathrm{m}}\left(C_{\mathrm{f}}-C_{\mathrm{m}}\right)\right]^{-1}
$$

et $I$ : matrice identité, $V_{\mathrm{f}}$ : taux volumique de fibre, $E_{\text {Esh }}$ : tenseur d'Eshelby, fonction de la forme et de l'orientation du renfort, $C_{\mathrm{m}}$ et $C_{\mathrm{f}}$ : tenseurs de rigidité de la matrice et du renfort.

L'utilisation du tenseur effectif de Ferrari [12] est mieux adaptée pour l'analyse des composites à fibres longues, c'est-à-dire pour des taux volumiques de fibres importants (> à $30 \%$ ). Son expression fait intervenir le terme $\left(1-V_{\mathrm{f}}\right)$ à la place de $V_{\mathrm{f}}$ dans l'équation (2).

Diverses expressions du tenseur de Eshelby en fonction de la forme du renfort (fibres courtes, fibres longues) sont données par Mura [13].

\section{L'homogénéisation}

Cette étape permet de déterminer le comportement équivalent du composite en donnant les relations entre les grandeurs locales (micro) et globales (macro). Les expressions des déformations et des contraintes macroscopiques sont les moyennes sur le volume $V$ du VER :

$$
\left\{\begin{array}{l}
E=\left(1-V_{\mathrm{f}}\right) \varepsilon_{\mathrm{m}}+\sum_{i=1}^{n} V_{\mathrm{f}}^{i} \varepsilon_{\mathrm{i}} \\
\sum=\left(1-V_{\mathrm{f}}\right) \sigma_{\mathrm{m}}+\sum_{i=1}^{n} V_{\mathrm{f}}^{i} \sigma_{\mathrm{i}}
\end{array}\right.
$$

d'où on peut déterminer l'expression générale des tenseurs de rigidité et de souplesse du composite en combinant les équations (1) et (4) par :

$$
\left\{\begin{array}{l}
C_{\mathrm{comp}}=C_{\mathrm{m}}+\sum_{i=1}^{n} V_{\mathrm{f}}^{i}\left(C_{\mathrm{i}}-C_{\mathrm{m}}\right) A_{\mathrm{i}} \\
S_{\mathrm{comp}}=S_{\mathrm{m}}+\sum_{i=1}^{n} V_{\mathrm{f}}^{i}\left(S_{\mathrm{i}}-S_{\mathrm{m}}\right) B_{\mathrm{i}}
\end{array}\right.
$$

On peut remarquer que si les tenseurs $A_{\mathrm{i}}$ et $B_{\mathrm{i}}$ sont égaux au tenseur identité $I$, nous retrouvons l'expression de la rigidité et de la souplesse respectivement du modèle de Voigt et de Reuss [1].

La rigidité homogénéisée du composite est obtenue en remplaçant le tenseur de localisation $A_{\mathrm{i}}$ dans l'équation (5) par son expression donnée par l'équation (2) :

$$
C_{\mathrm{comp}}=C_{\mathrm{m}}+\sum_{i=1}^{n} V_{\mathrm{f}}^{i}\left(C_{\mathrm{i}}-C_{\mathrm{m}}\right) A_{\mathrm{MT}}^{i}
$$

où $V_{\mathrm{f}}^{i}$ est la fraction volumique d'une famille de fibres. Dans l'étude d'un composite stratifié, $V_{\mathrm{f}}^{i}$ est égal au taux volumique de fibres de la couche considérée. L'homogénéisation micro est réalisée à l'échelle de la couche afin d'obtenir ses caractéristiques mécaniques.

Remarque : La fibre de carbone possède un comportement orthotrope au vu de son mode de fabrication. Son module de traction sens longitudinal est beaucoup plus élevé que ses modules sens travers. La matrice de rigidité de la fibre de carbone est alors déterminée en conséquence [11,13].

Les tableaux 1 et 2 présentent la comparaison des caractéristiques planes des composites obtenues expérimentalement et par le modèle théorique. Les valeurs finales données par le modèle ont été obtenues en plusieurs étapes.

La première donne les caractéristiques mécaniques de la résine (polyester et époxyde) incluant des porosités (3,85\% SMC-R ; 1,5 \% UD C/E). Le modèle de l'inclusion équivalente d'Eshelby est utilisé en entrant comme inclusion les porosités de forme sphérique (rapport de forme égal à 1) et de rigidité nulle.

La deuxième (pour le SMC-R seulement) donne (modèle de Mori-Tanaka) les caractéristiques mécaniques du mélange polyester, porosités et charge minérale (donnant les caractéristiques de la matrice) en fonction des taux volumiques et des caractéristiques mécaniques des constituants.

Enfin un dernier calcul permet d'obtenir les modules d'élasticité globaux à partir des rigidités de la matrice et des fibres (verre ou carbone), du rapport de forme des fibres et de la répartition spatiale des fibres (Tab. 3).

\subsection{Modèle d'endommagement}

Le modèle d'endommagement appliqué est celui introduit par Kachanov et Rabotnov en 1958, postulant que l'endommagement d'un matériau peut être décrit par ses 
Tableau 1. Tableau comparatif des caractéristiques mécaniques d'un composite SMC R $40 \%$.

\begin{tabular}{lccccccc} 
& $E_{x}(\mathrm{MPa})$ & $E_{y}(\mathrm{MPa})$ & $E_{z}(\mathrm{MPa})$ & $G_{x y}=G_{x z}(\mathrm{MPa})$ & $\nu_{x y}=\nu_{x z}$ & $G_{y z}(\mathrm{MPa})$ & $\nu_{y z}$ \\
\hline Modèle & 14720 & 14720 & 8832 & 3751 & 0,26 & 5550 & 0,33 \\
Expér. & 13450 & 12860 & - & 3322 & 0,248 & - & - \\
& \pm 260 & \pm 302 & & \pm 120 & $\pm 0,03$ & & \\
Ecart (\%) & 8,5 & 12,6 & - & 11 & 4,6 & & \\
\hline
\end{tabular}

Tableau 2. Tableau comparatif des caractéristiques mécaniques d'un composite unidirectionnel carbone époxy (T2H/.EH25).

\begin{tabular}{lccccccc} 
& $E_{11}(\mathrm{MPa})$ & $E_{22}(\mathrm{MPa})$ & $E_{33}(\mathrm{MPa})$ & $G_{12}=G_{13}(\mathrm{MPa})$ & $\nu_{12}=\nu_{13}$ & $G_{23}(\mathrm{MPa})$ & $\nu_{23}$ \\
\hline Modèle & 139000 & 9000 & 9000 & 5220 & 0,33 & 5550 & 0,43 \\
Expér. & 141000 & 8503 & - & 4810 & 0,31 & - & - \\
& \pm 2510 & \pm 152 & & \pm 80 & $\pm 0,02$ & & - \\
Ecart (\%) & 1,5 & 5,6 & - & 8 & 6,1 & - & - \\
\hline
\end{tabular}

Tableau 3. Forme et répartition spatiale des constituants des matériaux étudiés.

\begin{tabular}{lcccc} 
& Diam & Longueur & Rapport L/d & Répartition \\
\hline Charge minérale & $45 \mu \mathrm{m}$ & sphérique & 1 & diffuse \\
Porosité & $20 \mu \mathrm{m}$ & sphérique & 1 & diffuse \\
Fibre de verre & $10 \mu \mathrm{m}$ & $25 \mathrm{~mm}$ & 2500 & 1 orientation tous les $9^{\circ}$ \\
Fibre de carbone & $7 \mu \mathrm{m}$ & Infini & - & 1 orientation \\
\hline
\end{tabular}

effets sur les caractéristiques mécaniques du matériau et notamment les modules d'élasticité affaiblis par une variable scalaire $d[13-17]$.

La prise en compte de cet endommagement passe par la connaissance de l'évolution de la variable $d$ au cours du chargement. L'intérêt ici est d'appliquer à l'étude de nos deux matériaux, une méthodologie simple de prévision de l'endommagement (évolution de la variable $d$ ) et de la rupture (critère d'instabilité : test sur la variable $\dot{d}[15,16])$. Cette notion a été souvent abordée et appliquée aux composites stratifiés par Ladeveze $[13,17,18]$. On reprend dans notre étude le même formalisme.

L'évolution de l'endommagement est décrite par une approche thermodynamique classique dans le cadre des processus irréversibles. Le potentiel thermodynamique choisi est la densité volumique d'énergie libre $\rho \varphi$ dépendant des variables internes :

$$
\rho \varphi=\rho \varphi\left(\varepsilon^{\mathrm{e}}, d_{i}, T, V_{k}\right)=\frac{1}{2} C_{i j k l} \cdot \varepsilon_{i j}^{\mathrm{e}} \cdot \varepsilon_{k l}^{\mathrm{e}}
$$

où :

- $\varepsilon^{\mathrm{e}}$ est le tenseur des déformations élastiques,

$-d_{i}$ représente les variables d'endommagement,

- $T$ la température,

- $V_{k}$ d'autres variables telles que des variables d'écrouissage,

- $C_{i j k l}$ la matrice de rigidité du matériau,

- $\rho$ la densité du matériau.

L'énergie volumique de déformation élastique sous l'hypothèse des contraintes planes généralisées $\left(\sigma_{33}=0\right)$, est prise comme potentiel thermodynamique et s'écrit :

$$
2 W e=\frac{\sigma_{11}^{2}}{E_{11}}+\frac{\sigma_{22}^{2}}{E_{22}}-\frac{2 \nu_{12} \sigma_{11} \sigma_{22}}{E_{11}}+\frac{\sigma_{12}^{2}}{G_{12}}+\frac{\sigma_{13}^{2}}{G_{13}} \frac{\sigma_{23}^{2}}{G_{23}}
$$

Les variables associées à la déformation $\varepsilon^{\mathrm{e}}$ et à l'endommagement $d_{i}$ sont :

$$
\left\{\begin{array}{c}
\sigma\left(\varepsilon^{\mathrm{e}}, d_{i}\right)=\frac{\partial W e}{\partial \varepsilon_{\mathrm{e}}} \\
Y_{\mathrm{di}}\left(\varepsilon^{\mathrm{e}}, d_{i}\right)=-\frac{\partial W e}{\partial d_{i}}
\end{array}\right.
$$

\subsection{Variables d'endommagement}

L'endommagement est décrit par deux variables $d_{1}$ et $d_{2}$ à l'échelle du matériau dans le cas du composite à fibres courtes et de deux variables $d_{22}$ et $d_{12}$ à l'échelle du pli unidirectionnel dans le cas du composite stratifié. Ces variables sont appliquées aux différentes rigidités comme il est indiqué dans le tableau 4.

Compte tenu de l'isotropie transverse de la couche unidirectionnelle de carbone/époxy, les variables appliquées aux modules $G_{12}$ et $G_{13}$ sont identiques. Dans le cas du composite SMC-R, il a été vérifié que l'endommagement dans le plan est identique quelle que soit la direction; les fibres étant disposées aléatoirement [19]. L'endommagement appliqué aux modules de cisaillement $G_{12}$ et $G_{13}$ est identique.

Dans notre cas la variable taux de restitution d'énergie associée $Y_{\text {di }}$ est alors écrite de la manière suivante :

$$
Y_{\mathrm{di}}=\frac{1}{2} \frac{\sigma_{i}^{2}}{E_{i}^{0}\left(1-d_{i}\right)^{2}}
$$

où $i=(1,2)$ pour les composites à fibres courtes et $i=(12,22)$ pour les composites à fibres longues dans le repère du pli. $E_{i}^{0}$ représente les modules de traction et de cisaillement cités dans le tableau 4. 
Tableau 4. Récapitulatif des variables d'endommagements appliquées aux rigidités.

\begin{tabular}{|c|c|c|c|c|c|c|}
\hline $\begin{array}{l}\text { Module d'élasticité } \\
\text { du composite SMC } \\
\text { (repère global) }\end{array}$ & $E_{X}$ & $E_{Y}$ & $E_{Z}$ & $G_{X Y}$ & $G_{X Z}$ & $G_{Y Z}$ \\
\hline Variable d'endommagement & $d_{1}$ & $d_{1}$ & - & $d_{2}$ & $d_{2}$ & - \\
\hline $\begin{array}{l}\text { Module d'élasticité } \\
\text { du pli UD carbone/époxy } \\
\text { (repère couche) }\end{array}$ & $E_{11}$ & $E_{22}$ & $E_{33}$ & $G_{12}$ & $G_{13}$ & $G_{23}$ \\
\hline Variable d'endommagement & - & $\begin{array}{c}d_{22} \\
\text { si } \sigma_{22}>0\end{array}$ & - & $d_{12}$ & $d_{12}$ & - \\
\hline
\end{tabular}

La variable d'endommagement global définissant la limite du domaine de non fissuration est alors classiquement $[13,18]$ définie par l'expression suivante :

$$
Y_{\mathrm{g}}=\sup _{\tau \leq t} \sqrt{\sum k_{i} Y_{d i}}
$$

$k_{i}$ représente les termes de couplage des endommagements. Il est défini par le rapport des modules d'élasticité en traction et en cisaillement.

L'identification de l'évolution de $Y_{\mathrm{g}}$ en fonction des variables d'endommagement, est réalisée sur des essais de traction et/ou de cisaillement (méthode du rail) cyclés. L'identification des termes de couplages est aisée pour les composites unidirectionnels. Des essais de traction sur une stratification $[ \pm 65]$ et $[+45]$ permettent de les déterminer [13]. Concernant le composite SMC-R, il est nécessaire d'utiliser un essai de traction-cisaillement. L'essai Arcan a été utilisé [20] en appliquant un cycle de charge-décharge jusqu'à rupture. Cet essai permet de solliciter une éprouvette de forme « diabolo» sous une sollicitation mixte traction-cisaillement où le rapport de la contrainte de traction sur la contrainte de cisaillement peut varier. Le montage Arcan a été modifié par l'introduction de guide entre les mors, permettant ainsi de minimiser l'instabilité lors de décharges mécaniques. La configuration utilisée sur l'essai est celle pour un angle de $45^{\circ}$ afin d'obtenir un rapport de contrainte égale à 1,3.

Lors de chaque cycle de charge-décharge obtenu lors des essais, les valeurs de $Y_{d i}$ et $d_{i}=1-E_{i} / E_{0}[13,15,18]$ sont déduites. Ces évolutions sont données figures 4 et 5 pour les deux matériaux étudiés.

Ces évolutions permettent de déterminer le seuil d'endommagement $Y_{\mathrm{g}}(0)$ pour chaque matériau.

\subsection{Couplage endommagement pseudo plasticité}

La plasticité est utilisée pour retranscrire les déformations résiduelles lors de décharges mécaniques. Elle est induite par la plasticité de la matrice et les glissements aux interfaces fibres/matrice, mais aussi par certains blocages de fissures lors des décharges. On parlera

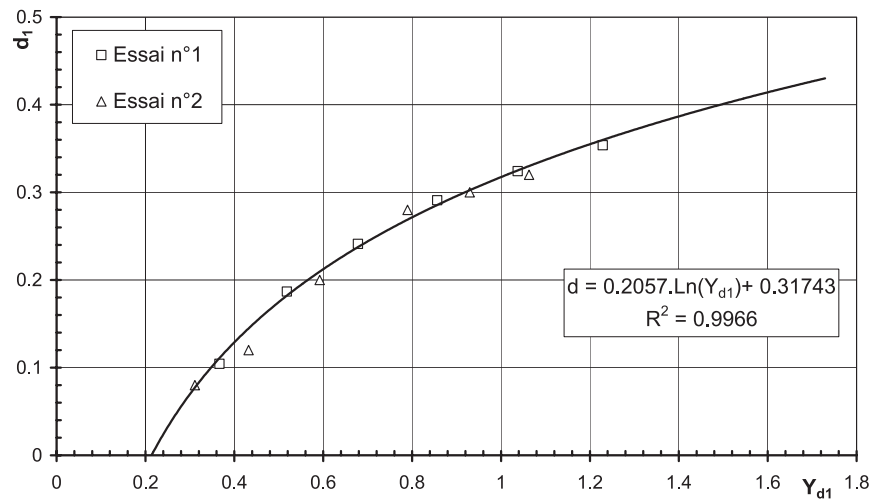

(a)

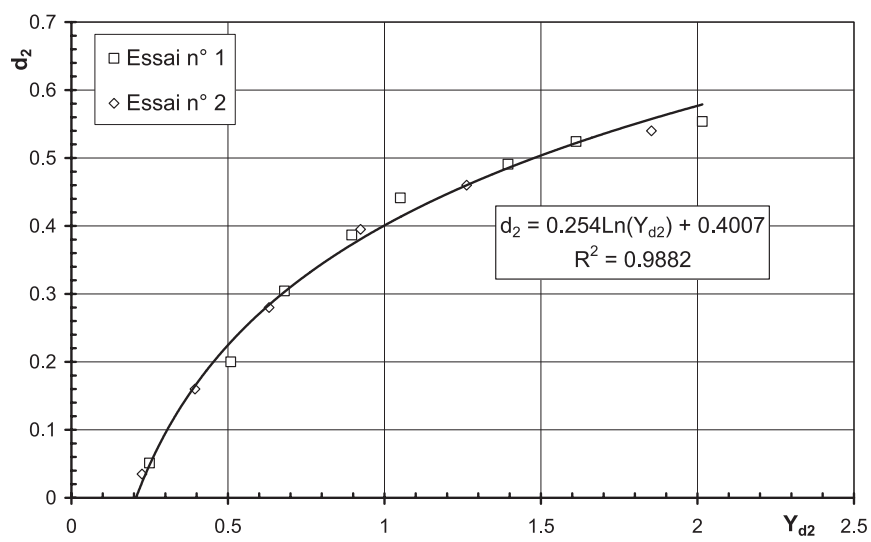

(b)

Fig. 4. (a) Endommagement en traction du composite SMCR. (b) Endommagement en cisaillement du composite SMC-R.

alors de pseudo-plasticité. Les lois classiques de plasticité en petites transformations sont :

- La déformation totale donnée par la somme d'une déformation élastique et d'une déformation permanente :

$$
\varepsilon=\varepsilon^{\mathrm{e}}+\varepsilon^{\mathrm{p}}
$$

- L'énergie libre $\varphi=\varphi\left(\varepsilon^{\mathrm{e}}, p, \alpha\right)$ où $p$ est la variable d'écrouissage isotrope, $\alpha$ la variable d'écrouissage cinématique. 


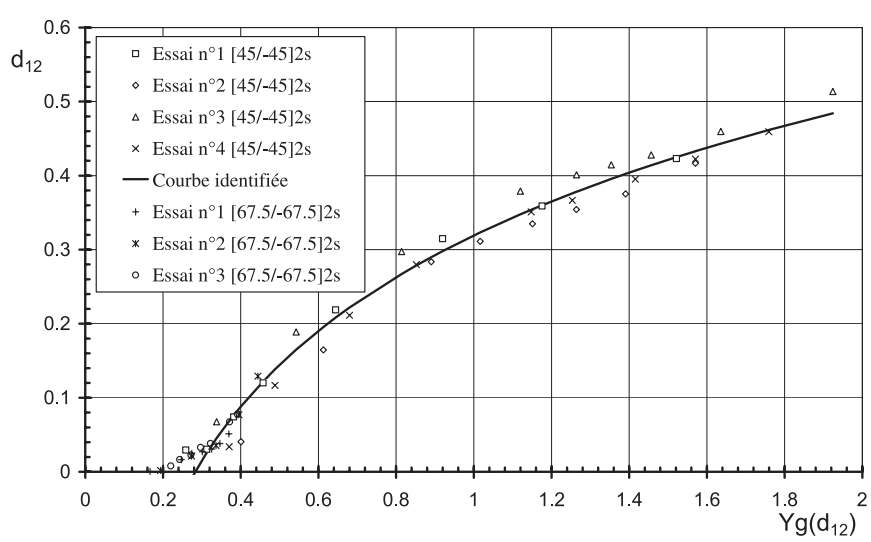

(a)

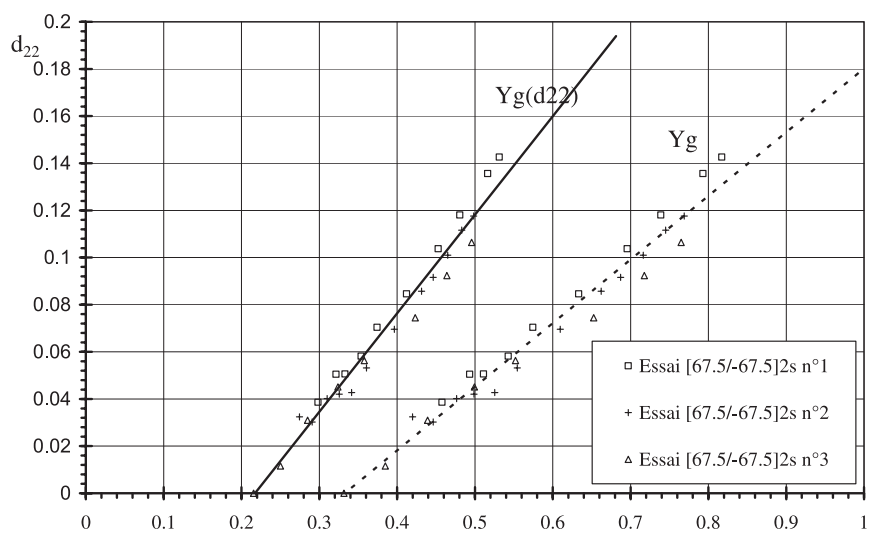

(b)

Fig. 5. (a) Endommagement en cisaillement du composite stratifié. (b) Endommagement en traction transverse.

- Les variables associées

$$
R=\rho \frac{\partial \varphi}{\partial p} ; X=\rho \frac{\partial \varphi}{\partial \alpha}
$$

- La surface de charge $f=f(\sigma, R, X)$ et l'hypothèse d'existence du multiplicateur plastique $\dot{\lambda}$ déterminé par la condition $f=\dot{f}=0$ avec les lois complémentaires associées

$$
\dot{\varepsilon}^{\mathrm{p}}=\dot{\lambda} \frac{\partial f}{\partial \sigma} \quad \dot{p}=-\dot{\lambda} \frac{\partial f}{\partial R} \quad \dot{\alpha}=-\dot{\lambda} \frac{\partial f}{\partial X}
$$

La variable $R$ décrit l'évolution de la taille de la surface de charge. La variable tensorielle $X$ décrit le déplacement de la surface de charge $[14,18]$. Le couplage des deux écrouissages peut être aussi réalisé. Les lois de normalité sont données par :

$$
\dot{\varepsilon}^{\mathrm{p}}=\frac{\dot{\lambda}}{f+R(p)+R_{0}} \frac{\sigma-X}{(1-d)^{2}} ; \quad \dot{p}=\dot{\lambda} ; \quad \dot{\alpha}=\dot{\varepsilon}^{\mathrm{p}}
$$

Les lois complémentaires s'écrivent :

$$
\dot{X}=\beta . \dot{\alpha}-\gamma \cdot X \cdot \dot{p} ; R=g(p)
$$

Le couplage plasticité-endommagement est réalisé de façon classique en introduisant la contrainte effective dans

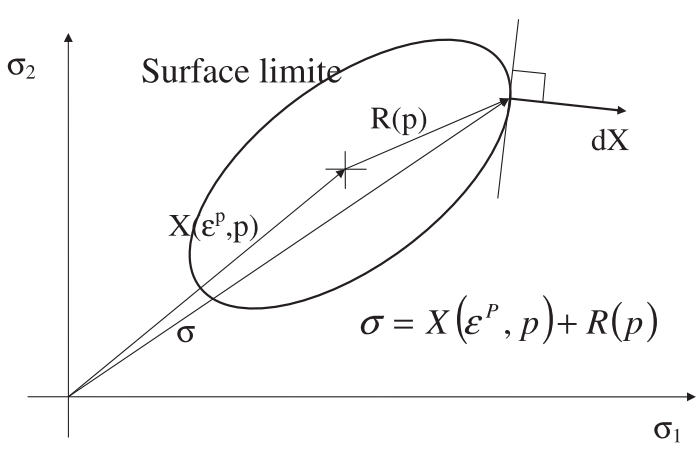

Fig. 6. Surface de charge.

le critère d'écoulement $[13,14]$. Le critère général de plasticité à écrouissage isotrope et cinématique non-linéaire utilisant un critère anisotrope s'écrit alors à la limite de l'écoulement :

$$
f=f(\tilde{\sigma}, R, X)=C(\tilde{\sigma}-X)-R(p)+R_{0}=0
$$

où $C(\tilde{\sigma}-X)$ est le critère définissant la forme de la surface de charge (Fig. 6) et $R_{0}$ le seuil initial d'écoulement.

Pour l'étude des composites SMC-R, le critère utilisé est le critère de Von Mises $\left(C(\tilde{\sigma}-X)=J_{2}(\tilde{\sigma}-X)\right)$. Cependant, lors du développement de l'endommagement, le critère définissant la surface de charge est modifié. Un critère anisotrope de type Hill devrait être utilisé.

Dans le cas du composite stratifié, le critère anisotrope utilisé $[13,18]$ s'écrit de la manière suivante :

$f(\sigma, X, R)=$

$$
\sqrt{\left(\frac{\sigma_{12}-X_{12}}{1-d_{12}}\right)^{2}+a^{2} \cdot\left(\frac{\sigma_{22}-X_{22}}{1-d_{22}}\right)^{2}}-R-R_{0}
$$

Les déformations plastiques sont nulles suivant la direction des fibres, identiques suivant les directions 12 et 13. Le coefficient de couplage $a^{2}$ est déterminé par essai et donné par le rapport entre les déformations plastiques. Les déformations transverses sont fonction du signe de la contrainte transverse : il n'y a pas de déformations résiduelles en compression transverse.

L'identification est réalisée par des essais en traction/cisaillement pour le composite SMC-R (essai Arcan [20]) et par des essais de traction sur des stratifiés $[ \pm 45]_{2 \mathrm{~S}},[45]_{8}$ et $[ \pm 65]_{2 \mathrm{~S}}$ pour l'unidirectionnel carbone/époxy $[13,16]$. Dans le cas d'un essai de traction, les variables de plasticité sont détaillées dans les deux paragraphes suivants.

\section{Cas de l'écrouissage isotrope}

Le domaine d'élasticité se modifie par dilatation. Les variables sont la déformation plastique cumulée $p$ et la variable associée $R$ définissant l'évolution de la surface de charge.

Dans ce cas, le critère de plasticité s'écrit :

$$
f=\frac{\sigma}{1-d}-\left(R+R_{0}\right)
$$




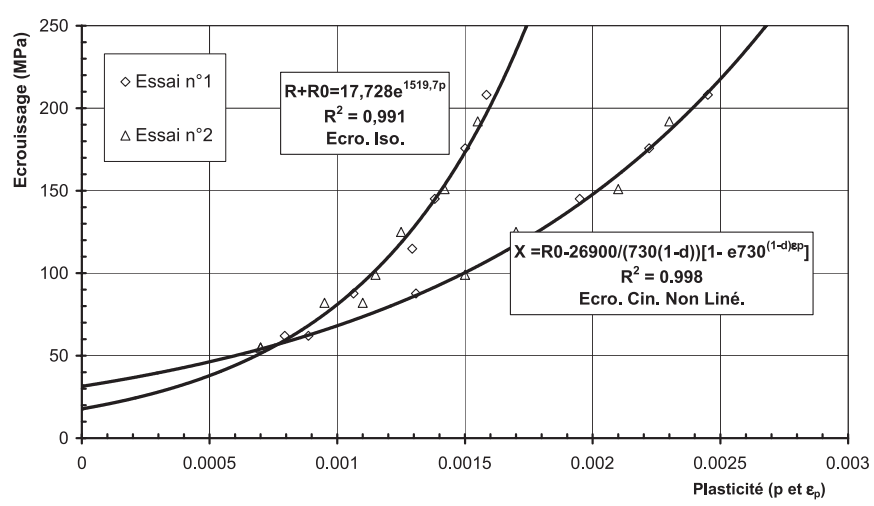

Fig. 7. Identification des paramètres de pseudo-plasticité pour SMC-R.

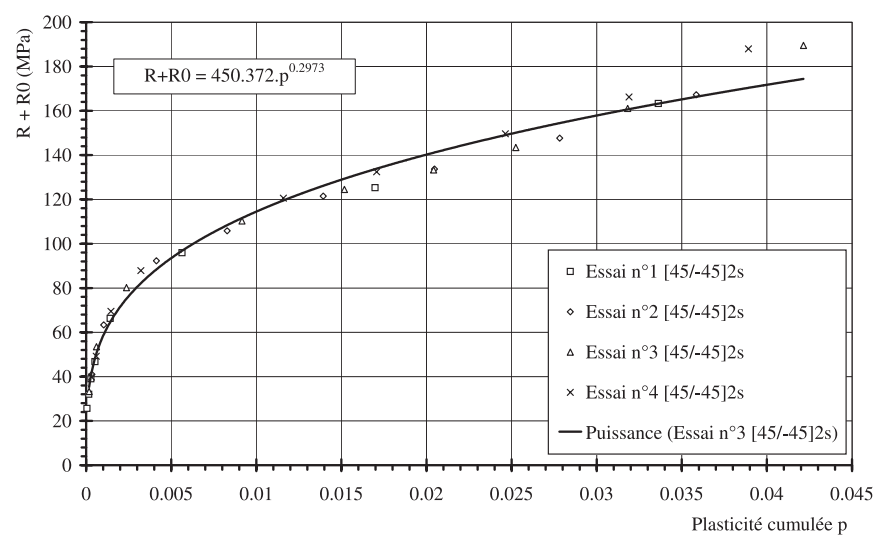

Fig. 8. Identification des paramètres de plasticité pour un UD carbone/époxy.

L'incrément de la déformation plastique cumulée est donné par :

$$
\dot{p}=\dot{\lambda}
$$

L'incrément de déformation plastique s'écrit :

$$
\dot{\varepsilon}^{\mathrm{p}}=\frac{\dot{\lambda}}{1-d}
$$

Les valeurs expérimentales sont relevées figures 7 et 8 . L'évolution de l'écrouissage est identifiée par une solution analytique où l'on peut relever le seuil initial de plasticité $R_{0}$.

\section{Cas de l'écrouissage cinématique non-linéaire} couplé à l'écrouissage isotrope

Le domaine d'élasticité se modifie par dilatation (variable $R$ ) et par déplacement (variable $X$ ). L'écrouissage cinématique non-linéaire a été couplé à l'écrouissage isotrope par l'écriture de la variable cinématique.

Le critère de plasticité s'écrit :

$$
f=\frac{\sigma-X}{1-d}-R_{0}
$$

L'incrément de la variable d'écrouissage est :

$$
\dot{X}=\beta(p) . \dot{\varepsilon}^{\mathrm{p}}-\gamma \cdot X \cdot \dot{p}
$$

La fonction $\beta(p)$ a été choisie de la forme :

$$
\beta(p)=\frac{\partial R(p)}{\partial p}
$$

L'incrément de déformation plastique s'écrit :

$$
\dot{\varepsilon}^{\mathrm{p}}=\frac{\dot{\lambda}}{1-d}
$$

En traction monotone, l'expression de $X$ (sans couplage) s'intègre analytiquement et s'écrit :

$$
X(0)=\frac{\beta(0)}{\gamma(1-d)}\left(1-e^{-\gamma(1-d) \varepsilon^{\mathrm{p}}}\right)
$$

Pour chaque incrément de charge, les valeurs de $X$, $\varepsilon^{\mathrm{p}}$ sont calculées. Les coefficients $\beta(0)$ et $\gamma$ sont alors déterminés (Fig. 7).

\section{Analyse numérique}

Le modèle développé ci-dessus a été implanté dans le module non-linéaire $\mathrm{MECANO}^{\circledR}$ du logiciel d'analyse numérique par éléments finis $\mathrm{SAMCEF}^{\circledR}$, par l'intermédiaire d'une routine écrite en Fortran. Le fichier d'entrée (jeu de données du modèle) est paramétré en terme de données d'endommagement et de plasticité. L'introduction des lois d'endommagement et de plasticité est réalisée par leur évolution analytique. La routine permet de donner en post-traitement l'évolution des déformations résiduelles, de l'endommagement élément par élément sous forme de courbe ou sous forme de cartographie. Ce processus est couplé à un algorithme de Newton-Raphson.

\subsection{SMC-R : analyse d'un essai de traction}

La validation du modèle a été réalisée sur un essai de traction. Les dimensions de l'éprouvette sont de $150 \mathrm{~mm}$ de longueur utile, $25 \mathrm{~mm}$ de largeur et $5 \mathrm{~mm}$ d'épaisseur. Le maillage est constitué de 30 éléments volumiques de degré 2. L'éprouvette est encastrée-libre. Les figures 9 et 10 montrent l'évolution de la contrainte appliquée en fonction de la déformation longitudinale pour les deux types d'écrouissage.

Les deux modèles sont très proches des résultats expérimentaux. L'écrouissage cinématique fait apparaître de faibles boucles d'hystérésis et retranscrit mieux les déformations résiduelles. Il ne semble pas primordial d'utiliser l'écrouissage cinématique, cependant, couplé à l'écrouissage isotrope, il permet de modéliser le durcissement cyclique observé.

La rupture globale de l'éprouvette est déterminée par l'instabilité de l'endommagement. 


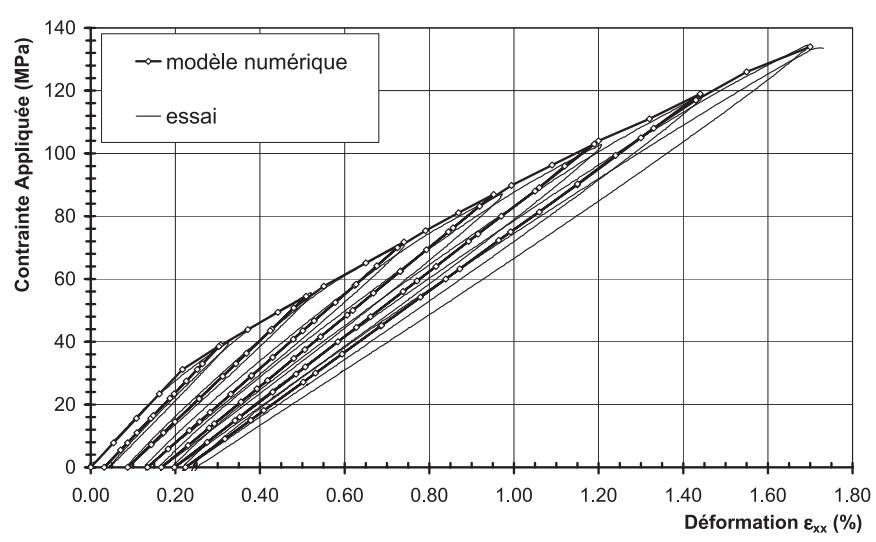

Fig. 9. Comportement expérimental/numérique : écrouissage isotrope; SMC-R.

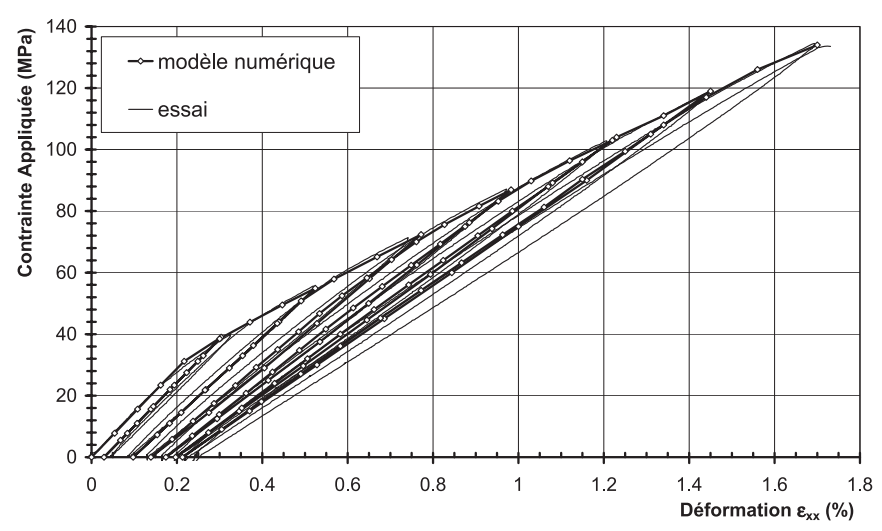

Fig. 10. Comportement expérimental/numérique : écrouissage cinématique + isotrope; SMC-R.

\subsection{UD carbone/époxy : analyse d'un essai de traction}

Deux stratifications sont utilisées pour les comparaisons expérimentales et numériques. La première permet de valider l'implantation de la pseudo-plasticité sur un essai à $[ \pm 45]_{2 \mathrm{~S}}$, la deuxième permet de valider le couplage entre l'endommagement en cisaillement et transverse $[ \pm 65]_{2 \mathrm{~S}}$. La pseudo-plasticité est pour ce matériau la pseudo-plasticité à écrouissage isotrope.

Les dimensions de l'éprouvette sont : $150 \mathrm{~mm}$ de longueur, $20 \mathrm{~mm}$ de largeur et $2 \mathrm{~mm}$ d'épaisseur. Le maillage est constitué de 60 éléments volumiques composites de degré 2 .

\section{Stratifié $[ \pm 45]_{2 S}$}

Les déformations résiduelles sont très bien retranscrites (Fig. 11), et ceci pour de très grandes déformations. La rupture de l'éprouvette est donnée par l'instabilité de l'endommagement et est obtenue pour une valeur de la variable d'endommagement égale à 0,5 comme déterminée par la théorie $[13,15]$. La différence de valeur à rupture entre l'expérimentation et le modèle vient du fait qu'il existe lors des essais, une réorientation des fibres à $45^{\circ}$, introduisant une contrainte à rupture surestimée.

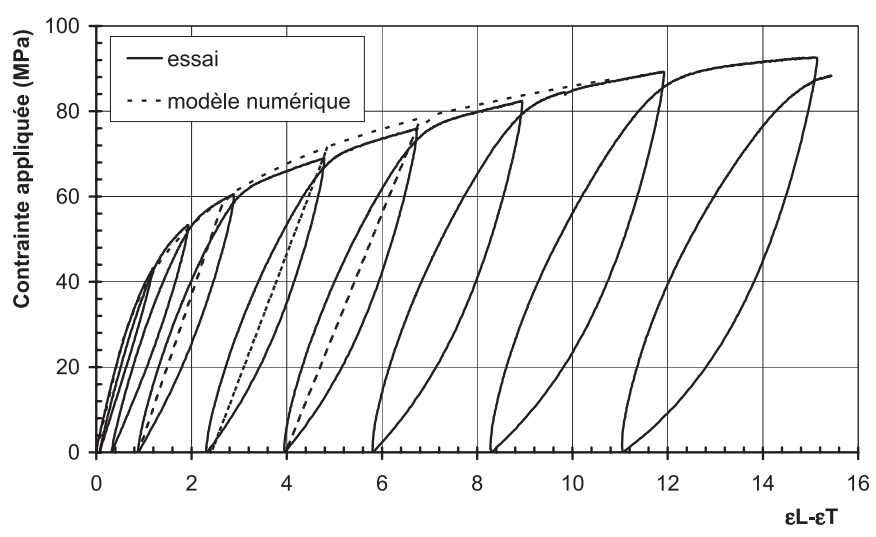

Fig. 11. Comparaison du comportement expérimental/numérique : essai sur stratifié $[ \pm 45]_{2 \mathrm{~S}}$.

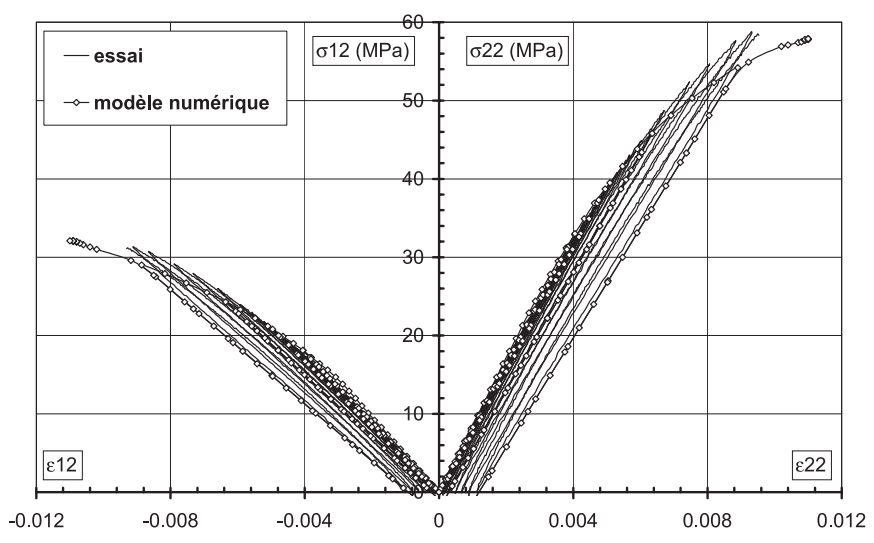

Fig. 12. Comparaison du comportement expérimental/numérique : essai sur stratifié $[ \pm 65]_{2 \mathrm{~S}}$.

\section{Stratifié $[ \pm 65]_{2 S}$}

Cet essai permet de coupler l'endommagement en cisaillement et en traction transverse. La figure 12 montre l'évolution des contraintes de cisaillement et transverse dans les plis à $65^{\circ}$. Le modèle est là encore assez proche des résultats expérimentaux et la prédiction de la rupture est bien restituée.

\subsection{Application au comportement de plaques trouées}

Afin de valider les modèles sur des structures plus complexes, des essais de traction sur éprouvettes trouées ont été menés. L'endommagement local en bord de trou est complexe et les modèles présentés doivent permettre de mieux appréhender le comportement en bord de trou de structures composites. La rupture numérique n'est pas abordée mais est en cours d'étude.

Les dimensions des éprouvettes trouées sont de $150 \mathrm{~mm}$ de partie utile, $30 \mathrm{~mm}$ de largeur, $5 \mathrm{~mm}$ d'épaisseur pour le composite SMC et $4 \mathrm{~mm}$ pour le composite stratifié. La séquence d'empilement du composite carbone/époxy est $\left[(90,45,0,-45)_{2}\right]_{\mathrm{S}}$; la couche à $0^{\circ}$ étant orientée dans le sens de l'effort. Le perçage de diamètre $5 \mathrm{~mm}$ a été réalisé à l'aide d'un foret spécifique. 


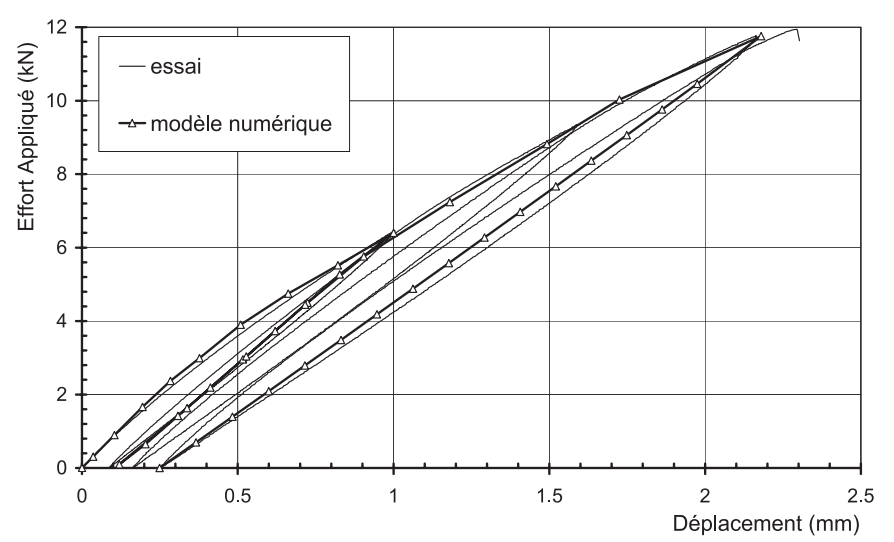

Fig. 13. Comportement expérimental/numérique d'un essai de traction sur éprouvette trouée (SMC-R).

Ce foret est un foret à coupe en bout nuance K20 utilisé pour l'usinage des composites à fibres de carbone [21].

La comparaison entre le comportement expérimental et le modèle numérique est réalisée jusqu'à la limite de la rupture expérimentale.

\subsubsection{SMC-R}

La figure 13 montre la comparaison des résultats expérimentaux et numériques en terme de comportement effort/déplacement pour le composite SMC-R. En analysant ces résultats, on constate que le modèle coïncide bien avec l'expérience. L'enveloppe du comportement est bien restituée ainsi que les déplacements résiduels.

Concernant l'endommagement, la figure 14, représente une radiographie $\mathrm{X}$ de l'éprouvette testée où l'on a superposé la cartographie numérique du module des endommagements sous forme d'iso-courbes. L'axe de sollicitation est vertical.

Les résultats numériques sont donnés à la limite de la rupture. L'endommagement maximal atteint 0,55 en bord de trou. Il est de 0,22 en partie courante de l'éprouvette.

La forme des endommagements donnée par le modèle est assez proche de l'expérience.

\subsubsection{Stratifié carbone/époxy}

La figure 15 montre la comparaison des résultats expérimentaux et numériques en terme de comportement effort/déplacement pour le composite stratifié carbone/époxy. Le modèle numérique donne là encore des résultats proches de l'expérience.

Le modèle permet d'indiquer les endommagements couche par couche. Ces endommagements sont superposés à une radiographie $\mathrm{X}$ (Fig. 16) sous formes d'iso courbes pour l'effort à rupture. L'axe de sollicitation est vertical.

Les valeurs maximales du module des endommagements en traction transverse et en cisaillement sont données (Fig. 16). Pour les plis à $90^{\circ}$ et à $45^{\circ}$, l'endommagement maximal (Figs. 16a et b) est situé en bord de

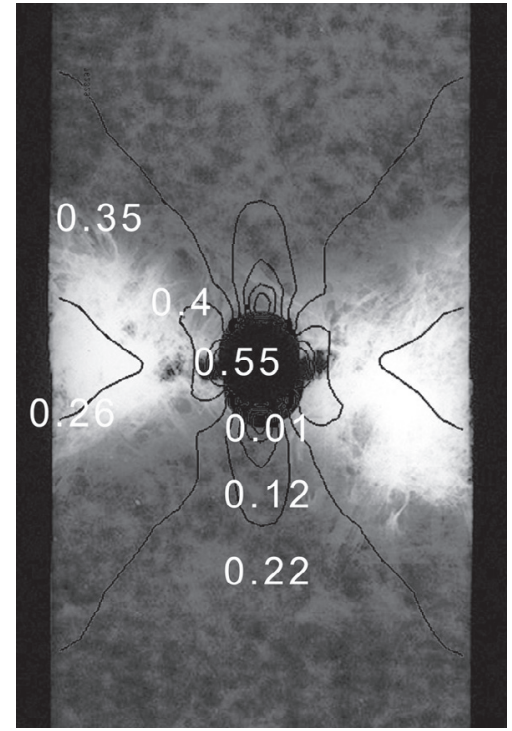

Fig. 14. Superposition de l'endommagement expérimental (RX) et de l'endommagement numérique (module) pour le composite SMC-R.

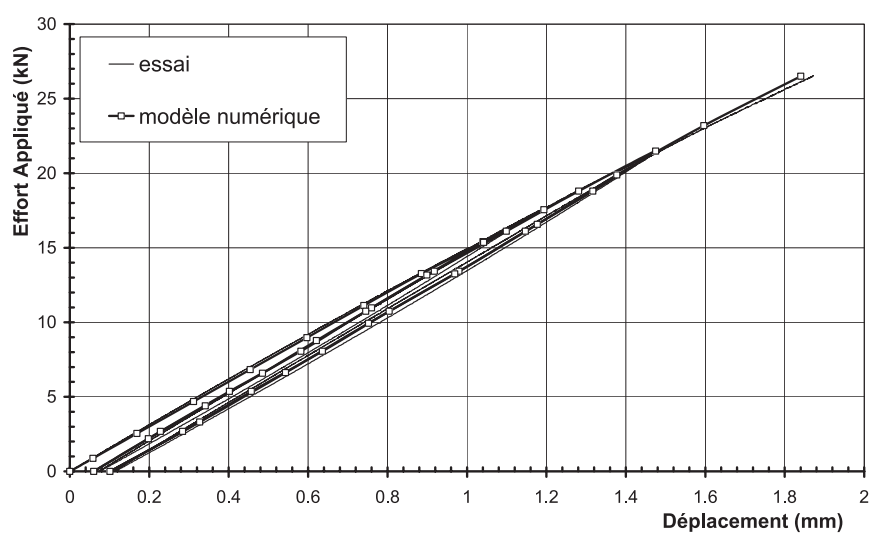

Fig. 15. Comportement expérimental/numérique d'un essai de traction sur éprouvette trouée (carbone/époxy quasi-iso).

trou sur une horizontale $\left(0,45\right.$ pour le pli à $90^{\circ} ; 0,38$ pour le pli à $45^{\circ}$ ). Il faut noter que la couche à $0^{\circ}$ s'endommage très localement à $45^{\circ}$ par rapport à l'axe de sollicitation (Fig. 16c).

Les résultats en terme de forme d'endommagement sont proches de ceux relevés par rayon $\mathrm{X}$.

\section{Conclusion}

Un modèle de comportement élastoplastique endommageable des composites a été proposé à l'aide d'une approche par la mécanique de l'endommagement. Un modèle micromécanique basé sur l'approche de Mori et Tanaka a permis de déterminer les caractéristiques mécaniques du composite mais aussi l'état de contrainte locale dans chaque constituant. Le modèle a été appliqué à un composite fibre-courte SMC-R et à un composite stratifié carbone/époxy. 


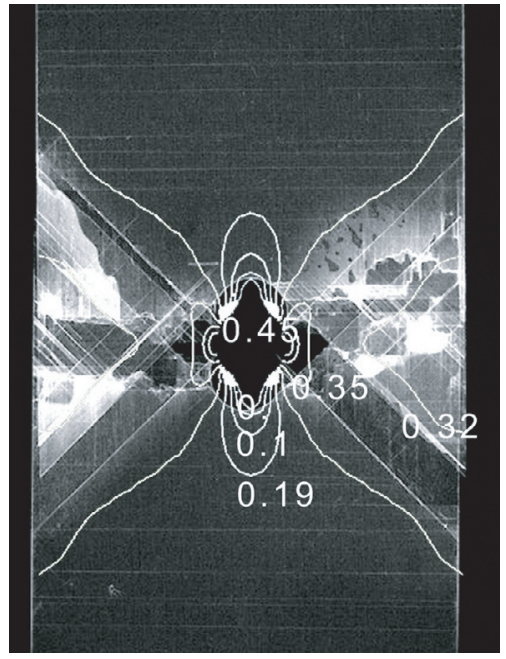

(a) Modèle : pli à $90^{\circ}$

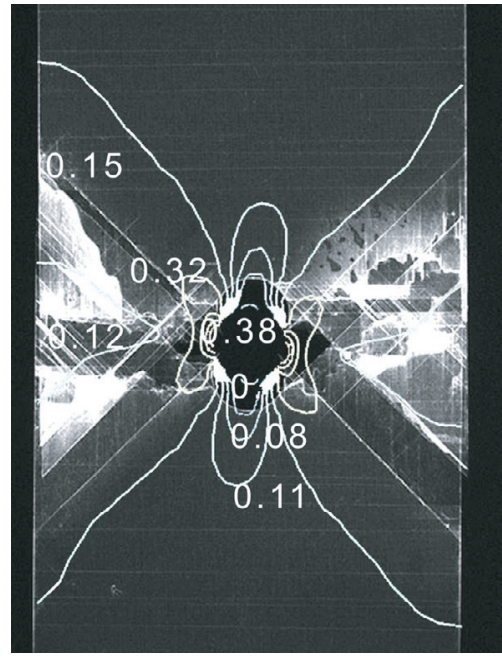

(b) Modèle : pli à $45^{\circ}$

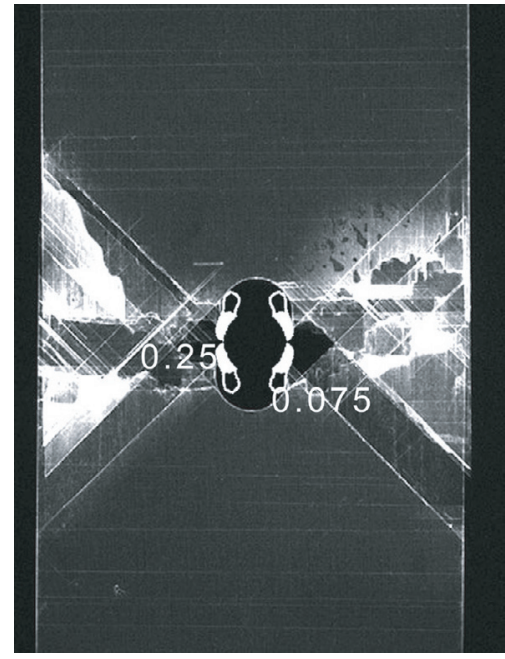

(c) Modèle : pli à $0^{\circ}$

Fig. 16. Superposition de l'endommagement expérimental (RX) et de l'endommagement numérique (module) pour le carbone/époxy quasi-isotrope.

La modélisation de l'endommagement progressif couplé à un critère de pseudo plasticité, fait apparaître une bonne corrélation essais-calculs pour des essais de traction. Le modèle de plasticité a été choisi comme un couplage entre l'écrouissage cinématique nonlinéaire et isotrope afin de modéliser le durcissement cyclique observé lors des essais sur composites fibres courtes. Pour les composites stratifiés, le couplage endommagement/plasticité à écrouissage isotrope, donne des résultats très proches des essais expérimentaux. Cependant la visco-plasticité n'a pas été prise en compte et est actuellement en développement.

Ces modèles ont été implantés dans un code de calculs par éléments finis à vocation industrielle ( $\left.\mathrm{SAMCEF}^{\circledR}\right)$. Bien qu'ils n'introduisent pas tous les phénomènes d'endommagement, leurs robustesses ont été validées.

\section{Références}

[1] D. François, A. Pineau, A. Zaoui, Comportement mécanique des matériaux : élasticité et plasticité, Édition Hermès, 1992

[2] J. Fitoussi, Étude micromécanique de l'influence de l'endommagement à l'interface fibre/matrice sur le comportement des composites organiques à renforts discontinus, thèse de l'ENSAM, Paris, 1995

[3] T. Mori, K. Tanaka, Average stress in matrix and average elastic energy of materials with misfitting inclusions, Acta Metallurgica 21 (1973) 571-574

[4] J.D. Eshelby, Elastic inclusions and inhomogeneities, Progress in Solid Mechanics 2, I.N. Sneddon, R. Hill (ed.), North-Holland, Amsterdam, 1961, pp. 89-140
[5] E. Haramburu, Approche intégrée du dimensionnement mécanique de structures en composite injecté avec fibres courtes : une interface entre injection et calcul de structure, thèse de l'Université Paul Sabatier, 2003

[6] J.M. Berthelot, Matériaux composites, comportement mécanique et analyse des structures, ed. Masson, Paris, 1992

[7] J. Fitoussi, N. Bourgeois, G. Guo, D. Baptiste, Prediction of the Anisotropic Damaged Behavior of Composite Materials : Introduction of Multi-Local Failure Criteria in a Micro-Macro Relationship, Computational materials science 5 (1996) 87-100

[8] C.L. Tucker, E. Liang, Stiffness predictions for unidirectional short-fibre composites: Review and evaluation, Composites science and technology 59 (1999) 655-671

[9] M. Bornet, T. Bretheau, P. Gilormini, Homogénéisation en mécanique des matériaux. Tome I (Matériaux aléatoires élastiques et milieux périodiques), Hermès, 2001

[10] F. Meraghni, F. Desrumaux, M.L. Benzeggagh, Implementation of a constitutive micromechanical model for damage analysis in glass mat reinforced composites structures, Composites Science and Technology 62 (2002) 2087-2097

[11] F. Desrumaux, F. Meraghni, M.L. Benzeggagh, Generalised Mori-Tanaka Scheme to Model Anisotropic Damage Using Numerical Eshelby Tensor, J. Comp. Mat. 35 (2001) 603-624

[12] A. Ferrari, Composite homogeneization via the polyinclusion approach, Compos. Engr. 4 (1994) 37-45

[13] T. Mura, Micromechanicals of defects in solids, Martinus Nijhoff Publishers, 1987

[14] P. Ladevèze, E. Le Dantec, Damage modelling of the elementary ply for laminated composites, Comp. Sci. Tech. 43 (1992) 257-267

[15] J. Lemaitre, J.L. Chaboche, Mécanique des matériaux solides, Édition Dunod, 1996 
[16] F. Lachaud, Délaminage des matériaux composites à fibres de carbone et à matrices organiques : étude numérique et expérimentale, suivi par émission accoustique, thèse de l'Université Paul Sabatier, 1997

[17] F. Lachaud, L. Michel, Étude de l'endommagement de matériaux composites carbones à matrice thermodurcissable et thermoplastique, Mécanique, Industrie et Matériaux 50 (1997) 52-57

[18] J.F. Maire, Vers une analyse unifiée et multiéchelle du comportement et de l'endommagement des matériaux composites, Comptes rendus des $11^{\text {e }} \mathrm{JNC}$, 1998, pp. 27-36

[19] C. Linhome, Un modèle de comportement élastoplastique endommageable pour les structures composites stratifiées, La recherche Aérospatiale 2 (1996) 119-135
[20] F. Lachaud, M. Bizeul, Analyse élastoplastique endommageable des composites à fibres courtes, $13^{\mathrm{e}}$ Journées Nationales sur les Composites (JNC-13), Strasbourg, 2003

[21] J.C. Devillers, D. Coutellier, E. Deletombe, D. Delsart, Détermination des caractéristiques d'interfaces d'un composite verre/époxy au moyen de l'essai Arcan, Matériaux 2002 de la conception à la mise en ouvre, Tours, 2002

[22] R. Piquet, B. Ferret, F. Lachaud, P. Swider, Experimental analysis of drilling damage in thin carbon/epoxy plates using special drills, Composites Part A: applied science and manufacturing, Elsevier, 2000, vol. 31, pp. $1107-1115$ 